\title{
A review of lifestyle weight management options in Scotland which meet best practice
}

\author{
W. Wrieden ${ }^{1}$ and A. Avenell ${ }^{2}$ \\ ${ }^{1}$ School of Pharmacy and Life Sciences, The Robert Gordon University, Aberdeen AB25 1HG, UK and ${ }^{2}$ Institute of Applied \\ Health Sciences, University of Aberdeen, AB25 2ZD, UK
}

The National Institute for Health and Clinical Excellence (NICE) ${ }^{(1)}$ has provided guidance for weight loss programmes recommended by primary care organisations and local authorities. These include aiming for a maximum weekly weight loss of $0.5-1 \mathrm{~kg}$ with a realistic target weight loss of 5-10\% of original weight, focusing on long-term lifestyle changes, addressing both diet (using a balanced healthy eating approach) and activity (recommending regular physical activity), including some behaviour change techniques such as keeping a diary, and recommending and/or providing ongoing support.

The aim of the review was to document the range of weight management programmes in Scotland that explicitly or implicitly follow NICE guidelines for best practice. It is estimated that 2.7 million adults in Scotland $\left(65 \%\right.$ of the adult population $\left.{ }^{(2)}\right)$ are now overweight or obese. The number of people recruited onto the programmes (within the last 2 years), or for commercial programmes which are ongoing, the maximum number of people attending weekly was requested to calculate an estimate of the 'reach', i.e. the percentage of the target population (overweight and obese) in an area.

The programmes included five commercial slimming programmes, and a range of programmes developed and/or delivered by health boards and community health partnerships. Most programmes stressed the importance of a healthy diet and incorporated some behaviour change techniques. The physical activity component of programmes varied from minimal to the incorporation of physical activity in all group sessions for the Rosemary Conley Diet and Fitness Clubs and Slimming NL. Most programmes run by health professionals were time limited, consisting of 6-12 sessions, with some offering an option for on-going sessions for review and monitoring. Only the commercial programmes were able to provide on-going support and often provided free membership for participants meeting and maintaining their target weight.

Some programmes such as Counterweight ${ }^{(3)}$, Weightwatchers ${ }^{(4)}$, Rosemary Conley ${ }^{(4)}$ and Slimming World ${ }^{(5)}$ have data to support their effectiveness, but most of the programmes are based on general evidence on weight management and have not been evaluated per se for effectiveness. The commercial organisations together treat approximately $1.6 \%$ of the overweight and obese population in Scotland each week and probably recruit in excess of 150000 people per year (i.e. a reach of 5\%). This compares with a reach of about $0.15 \%$ for Counterweight (January 2007 to November 2009) and 0.38\% for the Winning Weigh (NHS Tayside, January 2008 to September 2009).

These programmes alone would not make a significant impact on population levels of obesity. Policy interventions to address the environmental, cultural and social circumstances that lead to weight gain must be implemented. ${ }^{(2)}$.

W.W. acknowledges funding from Research Councils UK, AA from CSO of Scottish Government Health Directorates.

1. National Institute for Health and Clinical Excellence (NICE) (2006) Obesity: the prevention, identification, assessment and management of overweight and obesity in adults and children.

2. The Scottish Government (2010) Preventing Overweight and Obesity in Scotland: A Route Map Towards Healthy Weight. Edinburgh: The Scottish Government.

3. Ross H, Laws R, Reckless $\mathrm{J}$ et al. (2008) Evaluation of the counterweight programme for obesity management in primary care: a starting point for continuous improvement. Br J Gen Pract 58, 548-554.

4. Truby H, Baic S, deLooy A et al. (2006) Randomised controlled trial of four commercial weight loss programmes in the UK: initial findings from the BBC diet trials. BMJ 332, 1309-1314.

5. Lavin JH, Avery A, Whitehead SM et al. (2006) Feasibility and benefits of implementing a Slimming on Referral service in primary care using a commercial weight management partner. Public Health 120, 872-881. 